\title{
Treatment and management of migraine in neurological ambulatory practice in Poland by indicating therapy with monoclonal anti-CGRP antibodies
}

\author{
Izabela Domitrz ${ }^{1}$, Aurelia Lipa ${ }^{2}$ Jacek Rożniecki ${ }^{3}$, Adam Stępieńn ${ }^{4}$, Wojciech Kozubski ${ }^{5}$ \\ ${ }^{1}$ Department of Neurology, Faculty of Medical Sciences, Medical University of Warsaw, Warsaw, Poland \\ ${ }^{2}$ Medical Department, Teva Pharmaceuticals Polska Sp. z o.o., Warsaw, Poland \\ ${ }^{3}$ Department of Neurology, Stroke, and Neurorehabilitation, Medical University of Lodz, Lodz, Poland \\ ${ }^{4}$ Department of Neurology, Military Institute of Medicine in Warsaw, Warsaw, Poland \\ ${ }^{5}$ Department of Neurology, Charles Marcinkowski University of Medical Sciences, Poznan, Poland
}

\section{ABSTRACT}

Aim of study. To analyse Polish neurologists' familiarity with the diagnostic criteria for migraine, and how their methods of management of migraine work in daily practice.

Clinical rationale for study. Migraine is a common primary headache disease that causes substantial disability and reduces quality of life. Many migraine patients remain undiagnosed and deprived of treatment. Migraine treatment is problematic, and many patients discontinue preventive treatment, mainly because of a lack of efficacy or adverse effects. Antibodies targeting calcitonin gene-related peptide and its receptor seem to be effective and well-tolerated agents in migraine prevention.

Material and methods. This study was conducted using a computer-assisted web interview conducted with 51 neurologists in Poland, who agreed to participate in the study during a phone call. The questionnaire mainly assessed methods of treatment of migraine patients and diagnostic criteria used in neurological practice.

Results. Only one neurologist listed all of the diagnostic criteria for migraine, and $80 \%$ of physicians in their practice used only a part of the migraine diagnostic criteria, usually the migraine without aura criteria. On average, each neurologist had 55 patients under continuous care, seeing roughly 18 patients per month. On average, neurologists estimated that $77 \%$ of all patients with migraine had episodic migraine, whereas the rest had the chronic form. Importantly, $40 \%$ of patients with chronic migraine received all available preventive treatments without a satisfactory effect. Neurologists could offer monoclonal antibodies that target the CGRP-pathway (i.e. anti-CGRP and anti-CGRP receptor monoclonal antibodies) for the prevention of chronic migraine to about one in three patients with a chronic form of the disease.

Conclusions and clinical implications. Migraine is underdiagnosed and undertreated in Poland. Understanding of the diagnostic criteria for migraine among neurologists is insufficient. Most neurologists in Poland see patients in whom anti-CGRP/R-targeting treatment is indicated.

Key words: migraine, chronic migraine, anti-CGRP

(Neurol Neurochir Pol 2020; 54 (4): 337-343)

\section{Introduction}

Migraine is a common primary headache disease that causes substantial disability and seriously affects quality of life.
Globally, migraine is the second highest cause of years lived with disability, and it is the most common cause for referrals to a neurologist $[1,2]$. It is estimated that migraine affects $15-18 \%$ of women and $6 \%$ of men [3]. However, many people

Address for correspondence: Izabela Domitrz, Department of Neurology, Faculty of Medical Sciences, Medical University of Warsaw, Cegłowska 80 Str., 01-809 Warsaw, Poland, e-mail: izabela.domitrz@wum.edu.pl 
with migraine remain undiagnosed; about a quarter of patients with headaches meeting the diagnostic criteria for migraine do not receive a proper diagnosis [4]. In an online survey among Polish adults, 25\% had reported migraine symptoms within the previous 12 months, $2.5 \%$ had experienced migraine attacks with aura only, 6.4\% had experienced migraine attacks without aura, and $16.2 \%$ had experienced migraine attacks with or without aura. $37 \%$ of people with migraine declared that they had had migraine diagnosed by a physician in the past. $43 \%$ of people suffering from migraine attacks received medical advice for their condition; in the majority of cases they were referred to a primary care physician/general practitioner (71\%), or slightly less frequently to a neurologist (48\%) [5]. Similarly, in a large population study in Poland, $8.5 \%$ of participants had migraine; there are no other reliable studies except those quoted here [6].

In the USA, the National Hospital Interview Survey showed that the overall prevalence of migraine or severe headache was $15.3 \%$ [7]. Chronic migraine affects $1.4-2.2 \%$ of the general population, with a $2.5-6.5$-fold greater prevalence among women [8]. Compared to episodic migraine, chronic migraine poses a greater burden for patients and their families and generates three times more healthcare costs. In addition to frequent headaches, patients with chronic migraine often present anxiety, depression, obesity, and disorders of the respiratory, cardiovascular, and gastrointestinal systems [9].

The International Classification of Headache Disorders, 3rd. Edition (ICHD-III) defines chronic migraine as a headache occurring on 15 or more days per month for three months or longer, with features of a migraine headache on eight or more days per month [10]. Some have even suggested that having a migraine headache on eight or more days per month should itself be sufficient to define chronic migraine, because such patients have a disabling disease that requires effective treatment $[4,10,11]$.

There are three main aspects to managing migraine: lifestyle changes which include the recognition and avoidance of migraine triggers, the treatment of acute attacks, and preventive treatment. The most common migraine triggers include fasting, alcohol, caffeine withdrawal, or specific foods [12].

Effective treatment, acute and preventive, reduces the risk of progression and improves the outcome in patients with migraine [13].

Acute migraine should be treated pharmacologically, with nonsteroidal anti-inflammatory drugs, triptans, antiemetics, or, less commonly, ergots, all together being the first-line medications $[14,15]$. Preventive treatment should be considered in patients with chronic migraine or episodic migraine with frequent attacks, when the frequency or severity of headaches interferes substantially with work, school, or social life. Many drugs are used for preventive treatment, including $\beta$-blockers, calcium channel blockers, antidepressants, and anticonvulsants, as well as botulinum toxin, although this last-named is used exclusively in chronic migraine.
Moreover, because calcitonin gene-related peptide (CGRP) is crucially implicated in the pathogenesis of migraine, monoclonal antibodies that target CGRP or its receptor (CGRP-R) have been developed to prevent both chronic and episodic migraine $[16,17]$. On average, these agents shorten the duration of migraine by about a week per month [18]. The drugs for preventive treatment are chosen based on the headache pattern, comorbidities, medication tolerability, and patient preferences.

In practice, many patients discontinue classical/pharmacological preventive treatment, mainly because of a lack of efficacy or adverse effects. Thus, there is a need for highly tolerable and effective preventive treatments for migraine [19].

\section{Clinical rationale for this study}

Migraine is an underdiagnosed and undertreated disease in Poland. Greater knowledge in the field of migraine diagnosis will help neurologists estimate the number of patients who require treatment, including monoclonal antibodies that target the CGRP-pathway. The aim of this study was to analyse Polish neurologists' familiarity with the diagnostic criteria for migraine and their management preferences for migraine in daily practice, including the latest recommendations.

\section{Materials and methods}

In June and July 2019 we conducted a computer-assisted web interview with neurologists in Poland, who had agreed to participate in the study during a phone call.

The inclusion criteria were as follows:

- Specialisation in neurology (board certification)

- Seeing eight or more patients with migraine per month

- Seeing patients with chronic migraine

- Initiating treatment for chronic migraine

The neurologists filled out a questionnaire (spontaneous answer, open-ended question) that assessed:

- Knowledge of the diagnostic criteria for migraine

- Number of patients with episodic or chronic migraine under their care

- Treatment options of episodic and chronic migraine

- Preventive treatment for chronic migraine

- Need for new therapies in migraine

- Knowledge of anti-CGRP and/or anti-CGRP-R monoclonal antibodies.

\section{Results}

A total of 51 neurologists, with a mean experience in neurology of 17.2 years, completed the questionnaire. Only one neurologist ( $2 \%$ ) knew the exact definition of, and listed all the diagnostic criteria for, migraine without and with aura. Five $(\sim 10 \%)$ neurologists listed all the diagnostic criteria for migraine without aura, $80 \%$ of physicians in their practice 


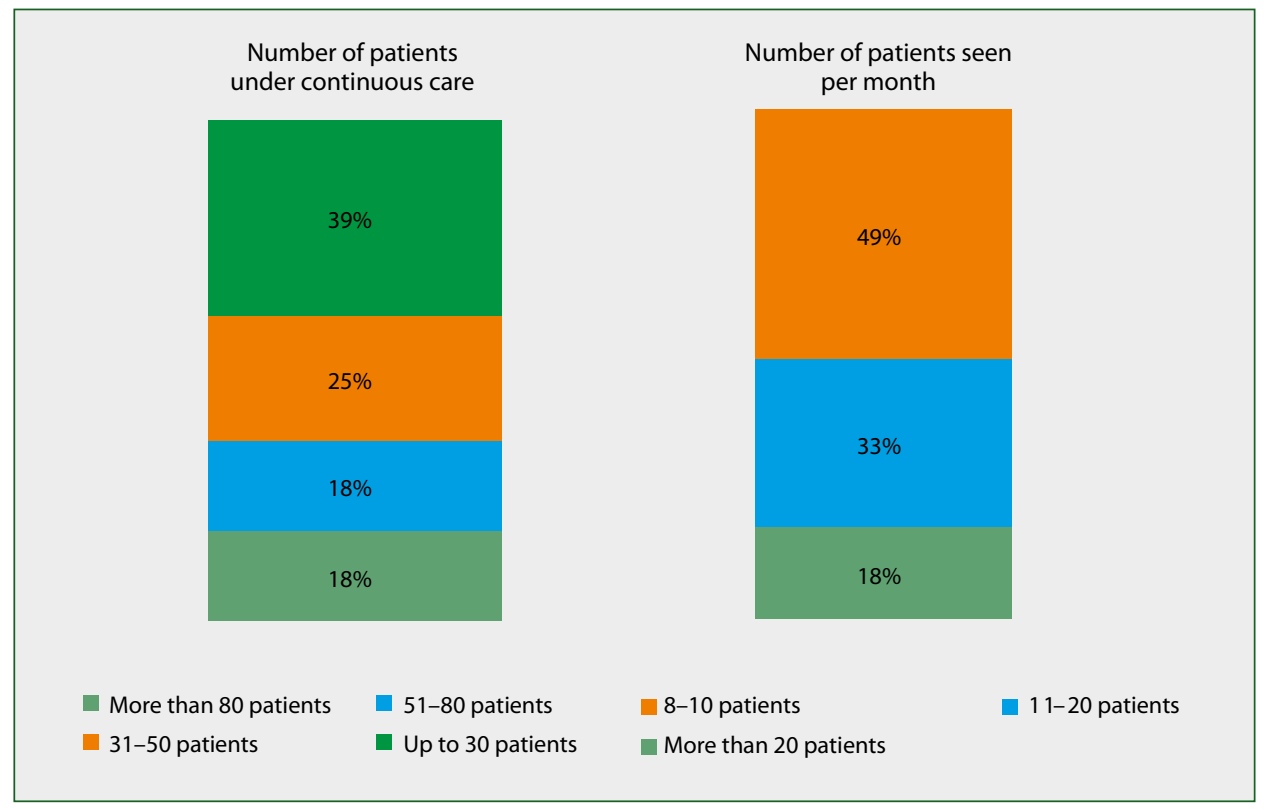

Figure 1. Percentages of neurologists declaring the number of patients under continuous care and the number of patients seen per month

used only a part of the migraine diagnostic criteria, usually migraine without aura criteria, and a large proportion of them ( $29 \%$ of neurologists) listed very limited criteria. Nine (18\% of neurologists) did not list any diagnostic criteria for migraine (Fig. S1).

Thirty-six (71\%) neurologists provided care to patients with migraine in a public outpatient clinic. Thirty-four (67\%) neurologists provided care for patients with migraine in hospital. Figure 1 shows the percentages of neurologists declaring the number of patients under continuous care and the number of patients per month. On average, each neurologist had 55 (median 40) patients under continuous care, seeing a mean of 18 (median 12) patients per month. On average, neurologists estimated that $77 \%$ of all patients with migraine had episodic migraine, whereas the rest had chronic migraine.

On average, neurologists estimated that $8 \%$ of patients with episodic migraine (which is one patient per month) required sick leave, with a mean absence of four days. Among patients with chronic migraine, $22 \%$ required sick leave (which is one patient per month), with a mean absence of seven days.

\section{Treatment for episodic migraine}

Each neurologist provided care to an average of $42 \mathrm{pa}-$ tients with episodic migraine. On average, $78 \%$ of patients with episodic migraine received prescription drugs, 19\% received over-the-counter (OTC) drugs only, and 3\% did not receive any drugs. Of patients who received pharmacological treatment, $53 \%$ received acute treatment only, $41 \%$ received preventive and acute treatment, and $6 \%$ received preventive treatment only (Fig. S2).

Thirty-four (67\%) neurologists initiated preventive therapy based on the number of days with migraine headaches in a month, with an average of seven days being sufficient to start treatment. Neurologists estimated that, among patients with episodic migraine, $36 \%$ overused OTC analgesics and $8 \%$ overused triptans.

\section{Treatment for chronic migraine}

On average, each neurologist provided care to 13 patients with chronic migraine. Most of these patients (84\%) received prescription drugs, $6 \%$ received OTC treatment ordered by a neurologist, $9 \%$ self-medicated with OTC drugs, and $1 \%$ did not receive any drugs. Most patients with chronic migraine (78\%) received preventive and acute treatment, $14 \%$ received acute treatment only, and $8 \%$ received preventive treatment only (Fig. 2).

Twenty-seven neurologists (53\%) initiated preventive treatment based on the number of days with a migraine headache in a month, with cut-off values ranging from three to 16 days. Eighteen of them initiated preventive treatment in their patients when the total length of time with a migraine headache in a month was 14-16 days (average $>12$ days). Fifteen (29\%) neurologists initiated preventive treatment based on the number of days with a headache in a month, and nine of them used the cut-off value of 14-15 days (average $>12$ days).

On average, of patients with chronic migraine on preventive treatment, $39 \%$ received anticonvulsants, $24 \%$ received $\beta$-blockers, and $22 \%$ received calcium channel blockers (Fig. S3). Among patients with chronic migraine, 54\% overused OTC analgesics and $18 \%$ overused triptans.

Twenty-nine (57\%) neurologists initiated preventive therapy for chronic migraine with anticonvulsants, $19(37 \%)$ with $\beta$-blockers, and 18 (35\%) with calcium channel blockers. First-line preventive treatment was effective in $64 \%$ of patients; 


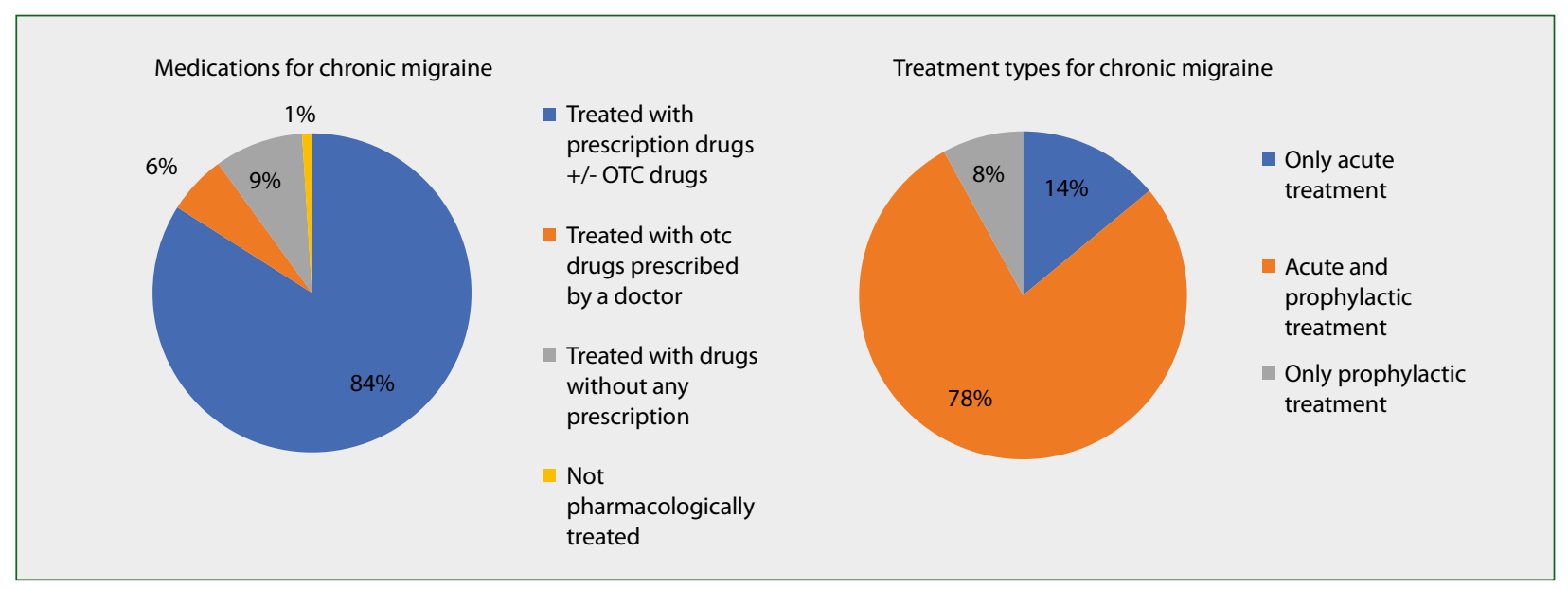

Figure 2. Medications and treatment types for chronic migraine

$28 \%$ of patients switched to a second-line drug, and $9 \%$ discontinued first-line treatment. Thirty-six (71\%) neurologists used anticonvulsants as second-line preventive treatment for chronic migraine. Second-line treatment was effective in 58\% of patients; $31 \%$ of patients switched to a third-line drug, and $11 \%$ of patients discontinued second-line treatment. Twenty-four (47\%) neurologists used anticonvulsants as third-line preventive treatment; botulinum toxin was prescribed by nine (18\%) neurologists. Third-line treatment was effective in $49 \%$ of patients; $32 \%$ of patients switched to a fourth-line drug, and 19\% discontinued third-line treatment. Twenty-seven (53\%) neurologists did not try fourth-line preventive treatment, and 36 (70\%) did not try fifth-line preventive treatments. Preventive treatment was discontinued due to a lack of effectiveness (34\% of patients), patient decision (30\%), or adverse effects (19\%). On average, each neurologist had about five (40\%) patients with chronic migraine, who had received all possible treatments in their opinion. On average, each neurologist could offer anti-CGRP/R-targeting treatment to 4.2 patients i.e. $33 \%$ of patients with chronic migraine, and $8 \%$ of all patients with migraine. Figure S4 shows the percentages of neurologists declaring the number of patients with chronic migraine who used all possible treatments and the number of patients who could be offered anti-CGRP or anti-CGRP-R treatment.

Forty-one (80\%) neurologists were aware that anti-CGRP and/or anti-CGRP-R monoclonal antibodies were available in Poland. Forty neurologists (78\%) knew that erenumab was approved in Poland. Four (8\%) neurologists declared that eptinezumab was available in Poland, which is not yet true. Nine (18\%) neurologists did not know of any anti-CGRP drugs approved in Poland (Fig. S5).

\section{Discussion}

In this study, only one neurologist knew the exact diagnostic criteria for migraine with and without aura. Less than a third of the responding neurologists listed some of the diagnostic criteria only for migraine without aura.

It must be underlined that the neurologists participating in this study defined themselves as being those who regularly manage migraine patients and are experts in this field.

Our findings show that knowledge of the diagnostic criteria for migraine among neurologists in Poland is very poor, which can lead to inappropriate treatment.

Our results are only partially in line with previous research, because in that previous research the problem was investigated only in relation to family doctors/primary care physicians. Gultekin et al. found that only one in 10 primary healthcare physicians was able to make a correct diagnosis of migraine [20]. In another study, $70 \%$ of patients with primary headache complaints did not receive a correct diagnosis from general practitioners [21]. It seems that educating doctors and patients on migraine symptoms is necessary in order to increase the recognition of migraine and to improve treatment outcomes, including the outcomes of preventive treatment.

In 2016, migraine was ranked as the number one cause of years lived with disability in people aged $15-49$, i.e. the sector of society who are the most occupationally active [22]. Patients with chronic migraine have considerably reduced health-related quality of life in physical, mental, and overall health aspects. The impact of migraine on life increases with the number of days on which migraine headaches occur [23].

Indeed, in the internet-based survey by Silberstein et al., headache-related disability, healthcare resource utilisation, and economic burden were found to increase gradually in patients presenting low-, moderate-, and high-frequency migraine, with the highest scores achieved in the chronic migraine subgroup [24].

Lack of effective treatment for migraine leads to frequent physician consultations, thereby generating substantial social and economic costs [25]. In 2017, the National Health Fund in Poland provided services for patients with migraine at 
a total cost exceeding 7 million PLN [26]. The costs due to absenteeism (nearly 100,000 days annually) were estimated at nearly 31 million PLN. According to the Social Insurance Institution in Poland, patients with episodic migraine have decreased productivity for 3.3 days quarterly, and those with chronic migraine, for 15.7 days quarterly. In this study, more than $20 \%$ of patients with chronic migraine required sick leave that lasted for an average of seven days.

Apart from the aforementioned absenteeism, presenteeism seems to be another, perhaps even more common and significant, problem. It is much more difficult to evaluate and to count the economic consequences of incomplete functioning in the workplace because of a set of symptoms of migraine attack including not only headache but also nausea, vomiting, hyperresponsiveness to light, sound, and smell, as well as avoiding any motor activities. Even though employees are physically at work, they are unable to concentrate, focus on work, and fully perform their duties. They do their jobs ineffectively, and are more likely to make mistakes.

It is clear that the burden of migraine, particularly chronic migraine, is underestimated.

In Poland, perhaps $1 \%$ of the general population might have chronic migraine, but fewer than half of those suffering from migraine receive medical help [26, 27].

Effective preventive treatment can reduce the economic costs of migraine. In practice, however, it is difficult to achieve effective migraine prevention. In this study, $40 \%$ of patients with chronic migraine received all available preventive treatments, but without a satisfactory result. The main reasons for treatment discontinuation were a lack of effectiveness (61\%) and adverse effects (41\%). In the International Burden of Migraine Study (IBMS-II), the reasons given for discontinuation were similar (lack of effectiveness $\sim 40 \%$; adverse effects $\sim 40 \%$ ). These findings indicate that there is a need for tolerable and effective preventive treatments for migraine [19].

The classic preventive oral treatments, including tricyclic antidepressants, $\beta$-blockers, calcium channel blockers, and antiepileptics, were not developed specifically for patients with migraine. In contrast, anti-CGRP-pathway monoclonal antibodies target CGRP or its receptor, a key neuropeptide which is implicated in the pathophysiology of migraine. Moreover, these medications (anti-CGRP-pathway monoclonal antibodies) are much better tolerated and have almost no contraindications.

With regard to the most serious adverse events due to some classic migraine prevention therapeutics, such as teratogenicity and liver toxicity, there is currently no evidence that anti-CGRP-pathway monoclonal antibodies would cause such adverse events [28]. The efficacy and safety of four anti-CGRP-pathway monoclonal antibodies have been assessed in groups of both episodic and chronic migraine patients [29-37].

In a phase 3 , randomised trial among patients with chronic migraine, the anti-CGRP monoclonal antibody fremanezumab, injected quarterly, reduced the number of headache days by $4.3 \pm 0.3$ per month and, when injected monthly, by $4.6 \pm 0.3$ per month, compared to $2.5 \pm 0.3$ per month in the placebo group $(\mathrm{p}<0.001$ for both comparisons with placebo). Injection-site reactions to the drug were common. The most common adverse event was injection-site pain, which occurred with comparable frequency in all three groups (30\%, $26 \%$, and $28 \%$, respectively) [29]. In the FOCUS study among 838 patients after the failure to up to four migraine preventive medication classes, fremanezumab monthly and quarterly reduced the number of days with migraine headache by 3.5 and 3.1 per month compared to placebo respectively $(\mathrm{p}<0.0001)$. Fremanezumab was well tolerated, and there were no safety concerns. The FOCUS study showed that patients with difficult-to-treat episodic or chronic migraine, who previously did not respond to up to four preventive medications, can achieve clinically meaningful improvement with fremanezumab [30].

Another anti-CGRP monoclonal antibody, galcanezumab, was assessed in prevention of episodic or chronic migraine in adult patients in three phase 3, randomised, double-blind, placebo-controlled studies: EVOLVE-1 [31], EVOLVE-2 [33], and REGAIN [34].

In the EVOLVE-2 trial, galcanezumab given to episodic migraine patients at a dose of $120 \mathrm{mg}$ or $240 \mathrm{mg}$ monthly reduced the number of days with migraine headache by $2.02 \pm 0.27$ and $1.90 \pm 0.27$, respectively, compared to placebo $(\mathrm{p}<0.001)$. Injection site pain was the most commonly reported treatment-emergent adverse event [33].

Erenumab, a monoclonal antibody targeting CGRP receptor, was assessed in patients with episodic migraine in the STRIVE trial. While significantly reducing the number of days with migraine (3.2 at a dose of $70 \mathrm{mg}$ and 3.7 at a dose of $140 \mathrm{mg}$ per month compared to 1.8 for placebo, $\mathrm{p}<0.001$ ), it showed a safety profile similar to that of the placebo [32]. It also proved to be efficacious and well tolerated in chronic migraine patients in a phase 2 clinical trial [35].

Eptinezumab, the only intravenous anti-CGRP monoclonal antibody, was investigated in the PROMISE-1 study in patients with episodic migraine [36], and in the PROMISE-2 study in patients with chronic migraine [37]. In both studies, it proved to be efficacious and well-tolerated.

In conclusion, all four anti-CGRP-pathway drugs appear to be highly successful in both episodic and chronic migraine management, while presenting favourable safety profiles.

Our study showed that each neurologist could offer anti-CGRP-pathway treatment for the preventive treatment of migraine to about four patients, i.e. about a third of patients with chronic migraine and about $10 \%$ of all patients with migraine. Of the four anti-CGRP-pathway monoclonal antibodies developed to date, three are approved in Europe/ Poland (approved first by FDI, next by EMA), i.e. erenumab, fremanezumab, and galcanezumab. Eptinezumab has not yet been approved in Poland or Europe, but it has received FDA approval [38]. About eight in 10 neurologists were aware that erenumab was available in Poland, but only $6 \%$ and $2 \%$ 
knew of the approval of galcanezumab and fremanezumab, respectively. During the study period, only erenumab was available in Poland; fremanezumab also became available in November 2019.

The cost of anti-CGRP-pathway monoclonal antibodies is considerably higher than that of other drugs used for migraine prevention. However, since monoclonal antibodies targeting CGRP and its receptor have demonstrated strong and consistent efficacy, favourable tolerability, and no safety signals, they can reduce the direct and indirect costs of migraine. Effectiveness, good tolerability, but also convenience of administration and, in our opinion, very high compliance and adherence, are evident advantages of anti-CGRP-pathway monoclonal antibodies. This kind of therapy may represent a valid option for episodic migraine and chronic migraine prevention in patients who are intolerant or who have inadequate response or contraindications to conventional preventive treatments [39]. Importantly, early treatment in patients with high-frequency episodic migraine may prevent progression from episodic to chronic migraine, thus reducing the burden on patients and society.

\section{Conclusions and clinical implications}

Migraine is underdiagnosed and undertreated in Poland. The diagnostic criteria for migraine used by neurologists in their practice are insufficient, mainly due to poor knowledge. Increasing migraine recognition will help to establish the number of patients who require treatment, including prevention. Importantly, $40 \%$ of patients with chronic migraine receive all available preventive treatments used not only for the prevention of chronic migraine but also for treating episodic migraine, but without a satisfactory result.

Anti-CGRP-pathway monoclonal antibodies are effective and have a favourable safety profile. Most neurologists in Poland see migraine patients in whom anti-CGRP-pathway treatment is both justified and indicated.

Ethical permission: Ethical approval was not necessary for the preparation of this article.

Funding: This research was funded by Teva Pharmaceuticals Polska Sp. $z$ o.o., Poland and was conducted in co-operation with PEX PharmaSequence, Poland.

We would like to thank all the physicians and interviewers involved in the research. Special thanks to Mrs. Katarzyna Proga, Mrs. Joanna Głażewska, and Mrs. Katarzyna Wróbel from PEX PharmaSequence.

Medical writing, professional editing, and language assistance was provided by Proper Medical Writing, Warsaw, Poland and sponsored by Teva Pharmaceuticals Polska Sp. z o.o., Poland. Conflicts of interest: A.L. is a Teva Pharmaceuticals Polska employee. The authors declare no conflicts of interest. All authors have read and approved the revised manuscript.

\section{References}

1. Goadsby PJ. Bench to bedside advances in the 21st century for primary headache disorders: migraine treatments for migraine patients. Brain. 2016; 139(Pt 10): 2571-2577, doi: 10.1093/brain/aww236, indexed in Pubmed: 27671024.

2. GBD 2016 Neurology Collaborators, GBD 2016 Headache Collaborators. Global, regional, and national burden of migraine and tension-type headache, 1990-2016: a systematic analysis for the Global Burden of Disease Study 2016. Lancet Neurol. 2018; 17(11): 954-976, doi: 10.1016/S1474-4422(18)30322-3, indexed in Pubmed: 30353868.

3. Goadsby PJ, Lipton RB, Ferrari MD. Migraine--current understanding and treatment. N Engl J Med. 2002; 346(4): 257-270, doi: 10.1056/ NEJMra010917, indexed in Pubmed: 11807151.

4. Tepper SJ, Dahlöf CGH, Dowson A, et al. Prevalence and diagnosis of migraine in patients consulting their physician with a complaint of headache: data from the Landmark Study. Headache. 2004; 44(9): 856-864, doi: 10.1111/j.1526-4610.2004.04167.x, indexed in Pubmed: 15447694.

5. Domitrz I, Lipa A, Rozniecki J, et al. Polish omnibus online survey on migraine conducted in a population of 2000 adults. Migrena news 2019;3(6):98-100, International Headache Congress Dublin 05-08; 2019.

6. Stępień A, Prusiński A, Suwała A. Selected epidemiological data on migraine prevalence in Poland. Ból. 2003; 3(4): 9-12.

7. Burch R, Rizzoli P, Loder E. The Prevalence and Impact of Migraine and Severe Headache in the United States: Figures and Trends From Government Health Studies. Headache. 2018; 58(4): 496-505, doi: 10.1111/head.13281, indexed in Pubmed: 29527677.

8. Natoli JL, Manack A, Dean B, et al. Global prevalence of chronic migraine: a systematic review. Cephalalgia. 2010; 30(5): 599-609, doi: 10.1111/j.1468-2982.2009.01941.x, indexed in Pubmed: 19614702.

9. Buse DC, Manack A, Serrano D, et al. Sociodemographic and comorbidity profiles of chronic migraine and episodic migraine sufferers. J Neurol Neurosurg Psychiatry. 2010; 81(4): 428-432, doi: 10.1136/ jnnp.2009.192492, indexed in Pubmed: 20164501.

10. The International Classification of Headache Disorders, 3rd edition. Cephalalgia. 2018; 38(1): 1-211.

11. Chalmer MA, Hansen TF, Lebedeva ER, et al. Proposed new diagnostic criteria for chronic migraine. Cephalalgia. 2020; 40(4): 399-406, doi: 10.1177/0333102419877171, indexed in Pubmed: 31544467.

12. Chądzyński P, Kacprzak A, Domitrz W, et al. Migraine headache facilitators in a population of Polish women and their association with migraine occurrence - preliminary results. Neurol Neurochir Pol. 2019; 53(5): 377-383, doi: 10.5603/PJNNS.a2019.0044, indexed in Pubmed: 31592536.

13. Charles A. The pathophysiology of migraine: implications for clinical management. The Lancet Neurology. 2018; 17(2): 174-182, doi: 10.1016/s1474-4422(17)30435-0.

14. Evers S, Afra J, Frese A, et al. European Federation of Neurological Societies, Members of the task force:. EFNS guideline on the drug treatment of migraine - report of an EFNS task force. Eur J Neurol. 2006; 13(6): 560-572, doi: 10.1111/j.1468-1331.2006.01411.x, indexed in Pubmed: 16796580.

15. Stępień A. Kryteria diagnostyczne i leczenie migreny w oparciu o obowiązujące zalecenia międzynarodowe. Med Dypl. 2011; 9(186): 81-87.

16. Monteith T. Chronic Migraine: Epidemiology, Mechanisms, and Treatment. Chronic Headache. 2018: 37-62, doi: 10.1007/978-3-319-91491-6_4. 
17. Weatherall MW. The diagnosis and treatment of chronic migraine. Ther Adv Chronic Dis. 2015; 6(3): 115-123, doi: 10.1177/2040622315579627, indexed in Pubmed: 25954496.

18. Ong JJ, Wei DYT, Goadsby PJ. Recent Advances in Pharmacotherapy for Migraine Prevention: From Pathophysiology to New Drugs. Drugs. 2018; 78(4): 411-437, doi: 10.1007/s40265-018-0865-y, indexed in Pubmed: 29396834.

19. Blumenfeld AM, Bloudek LM, Becker WJ, et al. Patterns of use and reasons for discontinuation of prophylactic medications for episodic migraine and chronic migraine: results from the second international burden of migraine study (IBMS-II). Headache. 2013; 53(4): 644655, doi: 10.1111/head.12055, indexed in Pubmed: 23458496.

20. Gültekin M, Balci E, İsmailoĞULLARI S, et al. Awareness of Migraine Among Primary Care Physicians in Turkey: A Regional Study. Noro Psikiyatr Ars. 2018; 55(4): 354-357, doi: 10.5152/npa.2016.19228, indexed in Pubmed: 30622393.

21. Kernick D, Stapley S, Hamilton W. GPs' classification of headache: is primary headache underdiagnosed? Br J Gen Pract. 2008; 58(547): 102-104, doi: 10.3399/bjgp08X264072, indexed in Pubmed: 18307854.

22. Steiner TJ, Stovner $\amalg$, Vos T, et al. Migraine is first cause of disability in under 50s: will health politicians now take notice? J Headache Pain. 2018; 19(1): 17, doi: 10.1186/s10194-018-0846-2, indexed in Pubmed: 29468450.

23. Blumenfeld AM, Varon SF, Wilcox TK, et al. Disability, HRQoL and resource use among chronic and episodic migraineurs: results from the International Burden of Migraine Study (IBMS). Cephalalgia. 2011; 31(3): 301-315, doi: 10.1177/0333102410381145, indexed in Pubmed: 20813784.

24. Silberstein SD, Lee L, Gandhi K, et al. Health care Resource Utilization and Migraine Disability Along the Migraine Continuum Among Patients Treated for Migraine. Headache. 2018; 58(10): 1579-1592, doi: 10.1111/head.13421, indexed in Pubmed: 30375650.

25. Wang SJ, Fuh JL, Huang SY, et al. Taiwan MAP Study Group. Diagnosis and development of screening items for migraine in neurological practice in Taiwan. J Formos Med Assoc. 2008; 107(6): 485-494, doi: 10.1016/S0929-6646(08)60157-6, indexed in Pubmed: 18583220.

26. Społeczne znaczenie migreny z perspektywy zdrowia publicznego i systemu ochrony zdrowia. Zakład Analiz Ekonomicznych i Systemowych Narodowy Instytut Zdrowia Publicznego - Państwowy Zakład Higieny. 2019: Warszawa.

27. Katarzyńska A, Domitrz I. Codzienny przewlekły ból głowy - historia, epidemiologia, klinika i przyszłość Neurologia i Neurochirurgia Polska. 2009; 43(2): 155-161.

28. Sacco S, Bendtsen L, Ashina M, et al. European headache federation guideline on the use of monoclonal antibodies acting on the calcitonin gene related peptide or its receptor for migraine prevention. J Heada- che Pain. 2019; 20(1): 6, doi: 10.1186/s10194-018-0955-y, indexed in Pubmed: 30651064.

29. Silberstein SD, Dodick DW, Bigal ME, et al. Fremanezumab for the Preventive Treatment of Chronic Migraine. N Engl J Med. 2017; 377(22): 2113-2122, doi: 10.1056/NEJMoa1709038, indexed in Pubmed: 29171818.

30. Ferrari MD, Diener HC, Ning X, et al. Fremanezumab versus placebo for migraine prevention in patients with documented failure to up to four migraine preventive medication classes (FOCUS): a randomised, double-blind, placebo-controlled, phase 3b trial. Lancet. 2019; 394(10203): 1030-1040, doi: 10.1016/S0140-6736(19)31946-4, indexed in Pubmed: 31427046.

31. Stauffer VL, Dodick DW, Zhang Qi, et al. Evaluation of Galcanezumab for the Prevention of Episodic Migraine: The EVOLVE-1 Randomized Clinical Trial. JAMA Neurol. 2018; 75(9): 1080-1088, doi: 10.1001/ jamaneurol.2018.1212, indexed in Pubmed: 29813147.

32. Goadsby PJ, Reuter U, Hallström Y, et al. A Controlled Trial of Erenumab for Episodic Migraine. N Engl J Med. 2017; 377(22): 2123-2132, doi: 10.1056/NEJMoa1705848, indexed in Pubmed: 29171821.

33. Skljarevski V, Matharu M, Millen BA, et al. Efficacy and safety of galcanezumab for the prevention of episodic migraine: Results of the EVOLVE-2 Phase 3 randomized controlled clinical trial. Cephalalgia. 2018; 38(8): 1442-1454, doi: 10.1177/0333102418779543, indexed in Pubmed: 29848108.

34. Detke HC, Goadsby PJ, Wang S, et al. Galcanezumab in chronic migraine: The randomized, double-blind, placebo-controlled REGAIN study. Neurology. 2018; 91(24): e2211-e2221, doi: 10.1212/ WNL.0000000000006640, indexed in Pubmed: 30446596.

35. Tepper S, Ashina M, Reuter U, et al. Safety and efficacy of erenumab for preventive treatment of chronic migraine: a randomised, double-blind, placebo-controlled phase 2 trial. Lancet Neurol. 2017; 16(6): 425-434, doi: 10.1016/S1474-4422(17)30083-2, indexed in Pubmed: 28460892.

36. Ashina M, Saper J, Cady R, et al. Eptinezumab in episodic migraine: A randomized, double-blind, placebo-controlled study (PROMISE-1). Cephalalgia. 2020; 40(3): 241-254, doi: 10.1177/0333102420905132, indexed in Pubmed: 32075406.

37. Lipton RB, Goadsby PJ, Smith J, et al. Efficacy and safety of eptinezumab in patients with chronic migraine: PROMISE-2. Neurology. 2020; 94(13): e1365-e1377, doi: 10.1212/WNL.0000000000009169, indexed in Pubmed: 32209650.

38. https://www.accessdata.fda.gov/drugsatfda_docs/ label/2020/761119s000lbl.pdf (22.02.2020).

39. Negro A, Martelletti P. Patient selection for migraine preventive treatment with anti-CGRP(r) monoclonal antibodies. Expert Rev Neurother. 2019; 19(8): 769-776, doi: 10.1080/14737175.2019.1621749, indexed in Pubmed: 31109209. 